\title{
Prevenção de Injúrias Causadas por Glyphosate em Soja RR POR MEIO dO USO DE AMINOÁCIDO ${ }^{1}$
}

\author{
Prevention of RR Soybean Injuries Caused by Exogenous Supply of Aminoacids
}

\author{
ZOBIOLE, L.H.S. ${ }^{2}$, OLIVEIRA JR., R.S. ${ }^{3}$, CONSTANTIN, J. ${ }^{3}$ e BIFFE, D.F. ${ }^{2}$
}

\begin{abstract}
RESUMO - A área de soja resistente ao glyphosate (RR) tem aumentado a cada ano. No entanto, essa expansão com a tecnologia da soja RR aumentou o uso de glyphosate significativamente, e muitos agricultores têm notado que alguns cultivares $R R$ apresentam injúrias visuais logo após a aplicação do glyphosate em pós-emergência. Dessa forma, dois experimentos foram instalados em anos distintos, com diferentes objetivos: o primeiro, visando avaliar a influência do glyphosate na soja RR nas variáveis fotossintéticas e de biomassa, e o segundo, a fim de reavaliar os mesmos parâmetros afetados na soja $R R$ pelo glyphosate, bem como o uso de diversas modalidades de aplicação de aminoácidos como forma de uma provável recuperação das plantas de soja com sua utilização exógena. A taxa fotossintética e o indice SPAD apresentaram decréscimos proporcionais às doses de glyphosate aplicadas, sendo o efeito pronunciado com a aplicação única desse herbicida. Devido ao decréscimo da taxa fotossintética, à produção de clorofila e à provável imobilização de nutrientes nos tecidos foliares causada pelo glyphosate, foi observada menor produção de biomassa. Entretanto, o uso de aminoácidos exógenos amenizou os efeitos indesejáveis desse herbicida na cultura da soja RR.
\end{abstract}

Palavras-chave: fotossíntese, herbicida, clorose, Rhizobium

\begin{abstract}
Glyphosate-resistant (RR) soybean crop areas have expanded every year. However, as a result of this expansion, the use of glyphosate has significantly increased, with the appearance of visual injuries in RR soybeans immediately after post-emergence application of the herbicide. Thus, two experiments were conducted in different years with different objectives. The first experiment aimed to evaluate the influence of glyphosate on photosynthetic variables and biomass production. The second experiment aimed to re-evaluate the same parameters affected in $R R$ soybeans by glyphosate, as well as the use of various methods of amino acid application, as a form of a likely recovery of the soybean plants following these exogenous applications. The photosynthetic rate and SPAD index decreased as the glyphosate rate increased, with a pronounced decrease after a single herbicide application. Overall, due to a decrease in the photosynthetic rate and chlorophyll production, as well as to a likely immobilization of shoot nutrient concentration by glyphosate, a significant biomass decrease was verified in the treatments with glyphosate application. However, the use of exogenous amino acids may be a strategy to safeguard the undesirable effects of this herbicide on $R R$ soybean.
\end{abstract}

Keywords: photosynthesis, herbicide, chlorosis, Rhizobium

Recebido para publicação em 12.2.2010 e na forma revisada em 18.2.2011.

2 Doutorando do curso do Programa de Pós-Graduação em Agronomia, Universidade Estadual de Maringá - UEM, Av. Colombo, 5790, Bloco J45, 87020-900 Maringá-PR, <lhzobiole@uol.com.br>; ${ }^{3}$ Professor Associado, Dep. de Agronomia, UEM. 


\section{INTRODUÇÃO}

No Brasil e no mundo, o aumento da área cultivada de soja resistente ao glyphosate (RR) é resultado do benefício dessa tecnologia no manejo de plantas daninhas, principalmente pelo seu amplo espectro de controle. Entretanto, alguns cultivares de soja RR apresentam injúrias após aplicação do glyphosate (Zablotowicz \& Reddy, 2007). No Brasil e na região centro-norte dos Estados Unidos, aplicações frequentes de glyphosate têm levado ao surgimento de deficiências de $\mathrm{Fe}, \mathrm{Zn}$ e $\mathrm{Mn}$ na soja RR (Huber, 2006; Johal \& Huber, 2009).

Em campo, o sintoma típico observado após a aplicação do glyphosate é conhecido como yellow flashing ou amarelecimento das folhas superiores. Esse sintoma tem ocorrido em áreas com adequada ou até alta fertilidade, como os Latossolos Vermelhos Eutroférricos, distribuídos pelas regiões norte e oeste do Paraná, e não somente em áreas com solos de baixa disponibilidade natural de micronutrientes, como os solos dos cerrados. Essa clorose na soja $R R$ pode ser atribuída à imobilização de cátions bivalentes, como Fe e Mn (Huber, 2006; Bott et al., 2008; Zobiole et al., 2010a). Como o glyphosate é considerado um ácido fosfônico (Franz et al., 1997) e esses ácidos possuem propriedades quelantes de cátions metálicos com cátios bivalentes e trivalentes (Kabachnik et al., 1974), essa é uma das possibilidades que explicariam a menor disponibilidade desses nutrientes nos tecidos das plantas (Zobiole et al., 2010a).

De acordo com Cakmak et al. (2009), a persistência desse sintoma clorótico depende da habilidade da planta de se recuperar pela absorção radicular dos elementos que foram imobilizados pelo glyphosate nos tecidos foliares. Para Reddy et al. (2004), esse sintoma indesejável na soja RR é atribuído ao acúmulo de AMPA (ácido aminometilfosfônico), primeiro metabólito fitotóxico do glyphosate, o qual é um dos responsáveis pela diminuição da biomassa seca da parte aérea e raiz e do teor de clorofila (Reddy et al., 2000; King et al., 2001; Zablotowicz \& Reddy, 2004). Em trabalhos anteriores (Zobiole et al., 2010a,c) ficou demonstrado que cultivares precoces foram mais suscetíveis à aplicação do glyphosate, com significativa redução no teor de clorofila, taxa fotossintética, concentração de nutrientes e biomassa seca da parte aérea e da raiz.

A ocorrência desse amarelecimento tem levado muitos produtores ao uso intensivo de fertilizantes foliares como alternativa para amenizar ou suprir essa possivel deficiência nutricional, sem, no entanto, haver necessariamente a recuperação da cultura. Outra possibilidade de amenizar os efeitos do glyphosate que tem sido aventada é o uso de bioestimulantes. Esses compostos são utilizados para aumentar o crescimento e a produtividade da soja, sob o argumento de que tais produtos podem aumentar a atividade microbiológica, biodisponibilidade de nutrientes e mineralização da matéria orgânica (Subler et al., 1998; Chen et al., 2002). Entretanto, no caso da soja RR não existem estudos específicos que comprovem sua recuperação no que se refere aos efeitos indesejáveis do glyphosate.

Pesquisas com herbicidas que agem no metabolismo de aminoácidos demonstram que o suprimento exógeno de aminoácidos pode reduzir a inibição do crescimento em plantas por eles atingidas (Jaworski, 1972; Gresshoff, 1979). Durante as décadas de 1980 e 1990, estudos visando descobrir os mecanismos de ação dos herbicidas eram feitos a partir da aplicação de determinadas moléculas em plantas e, posteriormente, incubando-as com diversas combinações de aminoácidos. Assim, com a reversão dos sintomas pela adição de aminoácido no meio de cultura, era possivel descobrir qual o sítio de ação do herbicida. Ray (1984) demonstrou que a adição de valina e isoleucina a herbicidas inibidores da acetolactato sintase (ALS), como o chlorsulfuron, reverteu completamente a inibição do crescimento de raízes de ervilha (Pisum sativum var. Alaska). Da mesma forma, com a adição desses mesmos aminoácidos, Shaner et al. (1984) observaram a reversão da inibição do crescimento de milho, causada pelo herbicida imazapyr.

No caso dos herbicidas inibidores da enzima 5-eno1-piruvil-3-shikimato-fosfato sintetase - EPSPs, um dos primeiros trabalhos conduzidos visando suprimir os efeitos do herbicida glyphosate pela adição da mistura de fenilalanina, tirosina e triptofano foi descrito por Jaworski (1972), o qual utilizou 
esses três aminoácidos para prevenir a inibição do crescimento de Lemma gibba e da bactéria Rhizobium japonicum pela aplicação do glyphosate. Esses mesmos resultados estão de acordo com os de Santos et al. (2005), para os quais a adição desses aminoácidos aromáticos ao meio de cultura preveniu a inibição do crescimento de $B$. japonicum, uma vez que este microrganismo possui a enzima sensivel ao glyphosate (Moorman et al., 1992). Da mesma forma, outros autores também relatam que a aplicação de aminoácidos aromáticos preveniu parcialmente a inibição do crescimento de raizes de pepino (Cucumis sativus cv. Wisconsin) causada pelo glyphosate e pelo ácido N-pirimidilaminometilenobifosfônico (Forlani et al., 1997).

O presente trabalho teve como objetivo avaliar, no primeiro experimento, a influência do glyphosate nas variáveis fotossintéticas e de biomassa na soja cv. BRS 242 RR. O segundo experimento foi conduzido visando reavaliar os mesmos parâmetros afetados pelo glyphosate na soja RR e também o uso de diversas modalidades de aplicação de aminoácidos como forma de levar a uma provável recuperação das plantas de soja com sua utilização exógena.

\section{MATERIAL E MÉTODOS}

Dois experimentos foram conduzidos na Universidade Estadual de Maringá, entre outubro de 2007 e dezembro de 2008 , em casa de vegetação $\left(23^{\circ} 25^{\prime} \mathrm{S}, 51^{\circ} 57^{\prime} \mathrm{W}\right)$ equipada com sistema evaporativo de refrigeração (25-35: $20-22{ }^{\circ} \mathrm{C}$ dia/noite), sob irradiância média de 700-1.200 $\mu \mathrm{mol} \mathrm{m} \mathrm{m}^{-2} \mathrm{~s}^{-1}$ durante período de luminosidade.

Sementes de soja foram esterilizadas por dois minutos em solução de $2 \% \mathrm{NaClO}$ e colocadas para germinar em rolo de papel (germilest). Plântulas com $5 \mathrm{~cm}$ de comprimento de raiz foram transplantadas para vasos contendo solução nutritiva. As unidades experimentais foram vasos de polietileno com 3,7 $\mathrm{dm}^{3}$, mantidos constantemente aerados. Para os primeiros 10 dias, as plantas foram mantidas em solução completa de Hoagland \& Arnon (1950), diluídas em 1/ 6 da concentração usual; nas duas semanas seguintes, foram cultivadas em solução diluída para $1 / 3$ e, após esse período, sob a concentração normal. As soluções foram trocadas a cada 10 dias e seu volume completado diariamente com água destilada e deionizada. $\mathrm{O} \mathrm{pH}$ das soluções foi mantido em 5,8 \pm 0,2 com adição de $\mathrm{NaOH}$ ou $\mathrm{HCl}$.

O primeiro experimento foi instalado visando avaliar a influência do glyphosate na soja RR, utilizando um esquema fatorial $(3 \times 2)+1$, com quatro repetições. Três doses de glyphosate (600, 1.200 e 1.800 g e.a. ha $\left.{ }^{-1}\right)$ foram combinadas com duas modalidades de aplicação (única e sequencial) para a soja RR (cv. BRS 242 RR) e um tratamento adicional sem aplicação do herbicida em sua isolinha parental não RR (cv. Embrapa 58). Aplicações da modalidade única foram realizadas no estádio V4 (24 DAE - dias após emergência), e aplicações da modalidade sequencial foram feitas no estádio V4 (24 DAE - 50\% da dose) e no estádio V7 (36 DAE - 50\% da dose) na soja $\mathrm{RR}$.

Os vasos foram colocados fora da casa de vegetação para aplicação dos tratamentos de glyphosate na formulação comercial de sal de isopropilamina (480 g i.a. $\mathrm{L}^{-1}$ ), utilizando um pulverizador costal com pontas SF110.02, sob pressão constante de $\mathrm{CO}_{2}\left(2 \mathrm{kgf} \mathrm{cm}^{-2}\right)$ e volume de calda de $190 \mathrm{~L} \mathrm{ha}^{-1}$. As condições ambientais durante as aplicações foram: temperatura do ar entre 25 e $29{ }^{\circ} \mathrm{C}$; umidade relativa do ar entre 80 e $89 \%$; velocidade do vento entre 5 e $10 \mathrm{~km} \mathrm{~h}^{-1}$; e céu aberto sem presença de nuvens. Após a aplicação do herbicida, os vasos retornaram para a casa de vegetação. As aplicações não causaram escorrimento superficial das folhas.

Um segundo experimento foi conduzido utilizando diferentes combinações de aplicação de aminoácidos com e sem glyphosate ( 1.800 g e.a. ha ${ }^{-1}$ ) no cv. BRS 242 RR e um tratamento adicional sem aplicação de herbicida e sem aminoácido em sua isolinha parental não RR (cv. Embrapa 58). Os tratamentos com glyphosate e com aminoácidos consistiram em mistura da solução de aminoácidos no momento da aplicação (V4 - $24 \mathrm{DAE})$ e após aplicação do glyphosate (V5 - 32 DAE; 7 DAA - dias após aplicação). Foi utilizada a dose de $2 \mathrm{~L} \mathrm{ha}^{-1}$, via aplicação foliar, do produto comercial AminoPlus ${ }^{\circledR}$, Ajinomoto, São Paulo, Brasil. Na sua composição esse produto apresenta: 
alanina: 1,164\%, arginina: 0,189\%, ácido aspártico: 1,943\%, ácido glutâmico: 3,316\%, glicina: $0,202 \%$, isoleucina: $0,171 \%$, leucina: $0,268 \%$, lisina: 0,240\%, fenilalanina: 0,143\%, serina: $0,179 \%$, treonina: $0,188 \%$, triptofano: 0,175\%, tirosina: $0,122 \%$, valina: $0,288 \%$; $11 \%$ e $\mathrm{K}_{2} \mathrm{O}: 1 \%$.

No experimento 1, quando as plantas atingiram o estádio R1 (aos $58 \mathrm{DAE}$ ) foram feitas avaliações da taxa fotossintética (micro mol $\left.\mathrm{CO}_{2} \mathrm{~m}^{-2} \mathbf{s}^{-1}\right)$ e índice SPAD, utilizando os equipamentos ADC model LCpro+ (Infra Red Gas Analyser, Analytical Development Co. Ltd, Hoddesdon, UK) e Minolta (SPAD-502 meter), respectivamente. As avaliações foram realizadas entre 7 e $11 \mathrm{~h}$, fazendo-se as medidas na parte mediana do segundo trifólio completamente expandido (folha diagnóstica). No segundo experimento, as avaliações foram realizadas imediatamente antes (V3, 22 DAE) e após (V7, 38 DAE) aplicação dos tratamentos, avaliandose taxa fotossintética $(A)$, taxa transpiratória $(E)$, condutância estomática $\left(g_{\mathrm{s}}\right)$ e índice SPAD na folha diagnóstica. Nesta mesma folha, um fluorômetro portátil (OS-30 - Opti-Sciences, Inc., Tyngsboro, MA) foi utilizado em pulsomodulado para determinar os parâmetros de fluorescência da clorofila $a$ : fluorescência inicial $\left(F_{0}\right)$, máxima fluorescência $\left(F_{\mathrm{m}}\right)$ e a razão da fluorescência: máxima fluorescência $(\mathrm{Fv} / \mathrm{Fm})$, empregando a equação: $\mathrm{Fv} / \mathrm{Fm}=(\mathrm{Fm}-$ Fo)/Fm (Genty et al., 1989). Diferentemente do primeiro experimento, no estádio R1 (54 DAE) do segundo experimento, foi avaliada a fluorescência das plantas. Após essa avaliação, em ambos os experimentos, as plantas foram separadas em parte aérea e raiz, embaladas em sacos de papel e secas em estufa de circulação forçada de ar a $65-70{ }^{\circ} \mathrm{C}$ até peso constante, quando foram tomados os pesos da biomassa seca da parte aérea (MSPA), raiz (MSRA) e total (MSTOTAL), por pesagem em balança de precisão.

Ambos os experimentos foram conduzidos em delineamento inteiramente casualizado, com quatro repetições. Os erros dos dados passaram pelos testes de Levene e ShapiroWilk, com o objetivo de avaliar a variância e a normalidade deles. No primeiro experimento, nas análises de regressão, foi escolhida a equação com maior grau de significância, com um máximo de segundo grau utilizando o programa estatístico SigmaPlot 10.0 (SPSS, 2000). No segundo experimento, os tratamentos foram comparados pelo teste de agrupamento de Scott-Knott a 5\% de probabilidade, utilizando-se o software SISVAR (Ferreira, 1999), e também com a isolinha parental não RR pelo teste de Dunnet a $5 \%$ de probabilidade pelo PROC GLM (SAS, 2001).

\section{RESULTADOS E DISCUSSÃO}

No experimento 1 , a taxa fotossintética (A) no estádio R1 (58 DAE) no tratamento sem glyphosate, para a soja cv. BRS 242 RR e sua isolinha parental não RR (cv. Embrapa 58), apresentou valores de 11,5 e $11,2 \mu \mathrm{mol} \mathrm{CO}_{2} \mathrm{~m}^{-2} \mathrm{~s}^{-1}$, respectivamente (Figura 1). Procópio et al. (2004) encontraram taxas similares (11 $12 \mu \mathrm{mol} \mathrm{CO} \mathrm{Cm}^{-2} \mathrm{~s}^{-1}$ ) aos $39 \mathrm{DAE}$ para Glycine max e Phaseolus vulgaris, as quais foram consideradas ótimas para essa fase vegetativa (Liu et al., 2005). Da mesma forma, Zobiole et al. (2010a) avaliaram os parâmetros fotossintéticos em diferentes estádios fenológicos da soja conduzida sob solução nutritiva em casa de vegetação e observaram, no estádio $\mathrm{R} 1$ para $\mathrm{cv}$. BRS $242 \mathrm{RR}$, valores entre $10 \mathrm{e}$ $11 \mu \mathrm{mol} \mathrm{CO} \mathrm{m}^{-2} \mathrm{~s}^{-1}$ nos tratamentos sem aplicação de glyphosate.

A taxa fotossintética e o índice SPAD apresentaram decréscimos proporcionais às doses de glyphosate aplicadas (Figura 1). Esses resultados estão de acordo com relatos feitos por agricultores, mostrando que alguns cultivares de soja RR são visualmente injuriados pelo glyphosate (Zablotowicz \& Reddy 2007). Em relação à modalidade de aplicação, ocorreu maior redução de $A$ com a aplicação única do que a aplicação sequencial, provavelmente pelo fato de que aos $24 \mathrm{DAE}$ (estádio V4) plantas que receberam aplicação única foram expostas a uma maior concentração do herbicida que aquelas que receberam $50 \%$ da dose em modalidade sequencial de aplicação. A relação entre $A$ e indice SPAD mostrou que o aumento da dose refletiu em diminuição de $A$ e do teor de clorofila, provavelmente pelo dano direto do glyphosate ao cloroplasto (Kitchen et al., 1981; Lee, 1981; Reddy et al., 2004) ou pela quelatização ou imobilização de cátions como Mg e $\mathrm{Mn}$, requeridos para a formação de clorofila e para a fotossintese, respectivamente (Beale, 1978; Taiz \& Zeiger, 1998). Em trabalhos 
anteriores (Zobiole, 2010a,b,c) também se observou maior injúria causada pelo glyphosate na modalidade de aplicação única, quando comparada à aplicação sequencial.
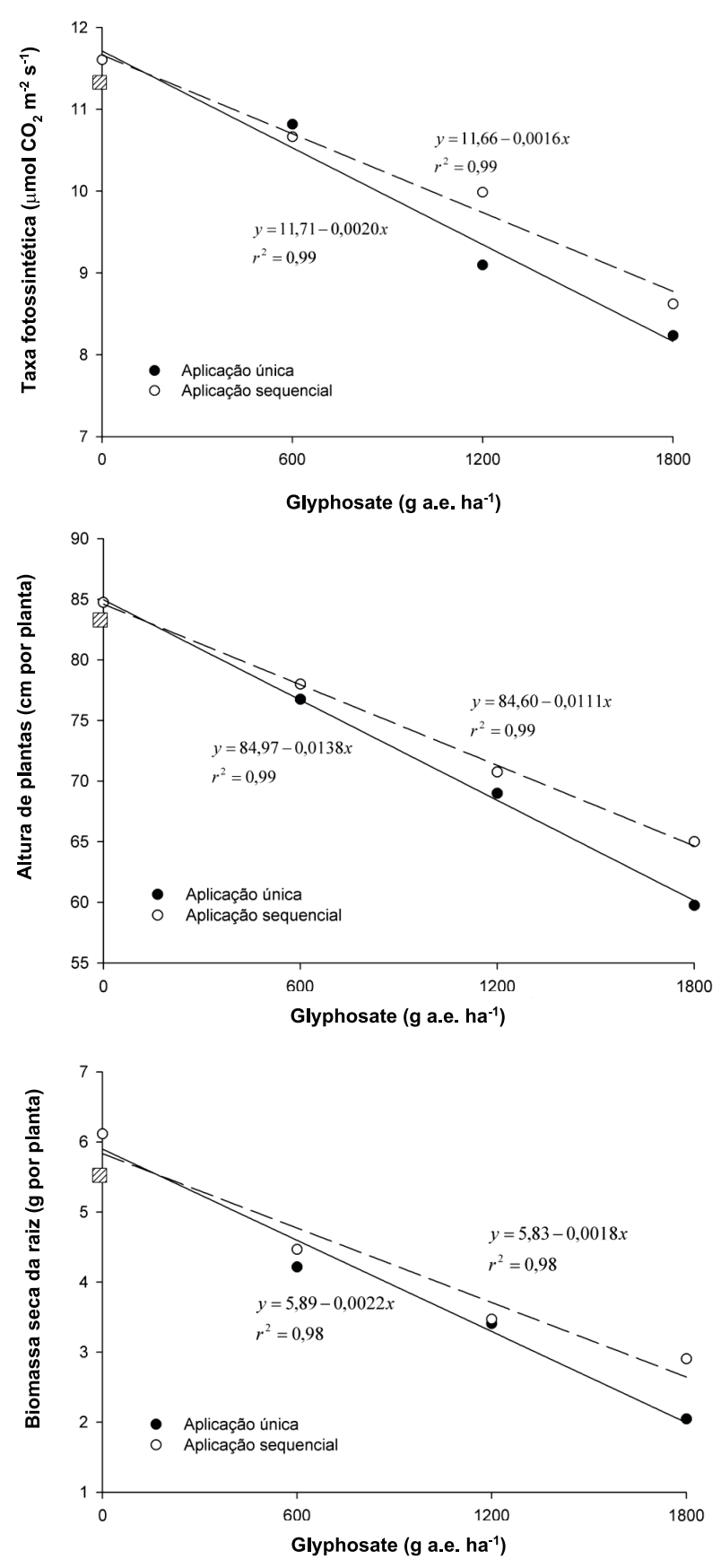

Estudos preliminares demonstraram que os parâmetros fotossintéticos $A$ e índice SPAD foram reduzidos pelo glyphosate em todos os grupos de maturação de cultivares de soja RR
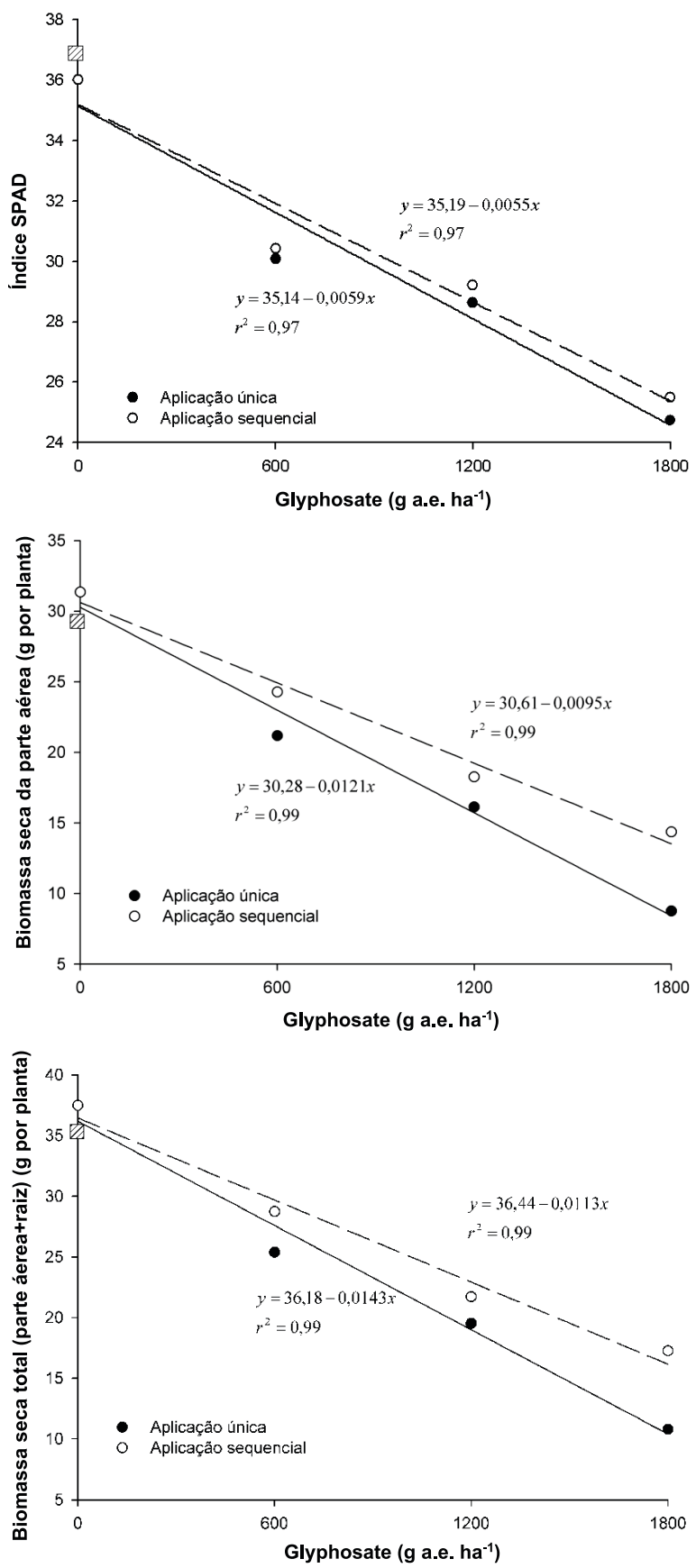

Z Isolinha parental não RR (cv. Embrapa 58).

Figura 1 - Taxa fotossintética, índice SPAD, altura de plantas, biomassa seca da parte aérea, da raiz e total por plantas de soja cv. BRS 242 RR submetidas a diferentes doses de glyphosate, em aplicação única (24 DAE) e sequencial (24 DAE - $50 \%$ dose e 36 DAE - 50\% dose), aos 58 DAE (experimento 1 ). 
avaliados em diferentes tipos de solos, porém não houve diferença entre os tratamentos sem glyphosate na soja RR e suas respectivas isolinhas parentais (Zobiole et al., 2010a,c). O mesmo resultado foi observado neste experimento quando se comparou o tratamento sem glyphosate para cv. BRS 242 RR e sua isolinha parental não RR (cv. Embrapa 58).

Devido ao decréscimo da taxa fotossintética e da produção de clorofila (Figura 1) causado pelo glyphosate (Zobiole et al., 2010a) e, provavelmente, pela imobilização de nutrientes nos tecidos foliares (Cakmak et al., 2009), foram observadas menor altura de plantas e menor produção de biomassa seca da parte aérea, da raiz e, consequentemente, da biomassa total (Figura 1). De acordo com Ireland et al. (1986), qualquer substância química que induz a alterações no metabolismo da folha, direta ou indiretamente, afeta o nivel dos intermediários e/ou da atividade das enzimas do ciclo de Calvin. Como a biomassa total da soja depende da energia suprida pela fotossintese para sintetizar compostos carbônicos (Shibles \& Weber, 1965), diminuições na assimilação do $\mathrm{CO}_{2}$ levam à redução na biomassa e no acúmulo de carboidratos (Magalhães Filho et al., 2008).

Outros autores têm atribuído a redução de biomassa causada pelo glyphosate na soja RR ao acúmulo do ácido aminometilfosfônico (AMPA) - seu principal metabólito (Reddy et al., 2004). Estudos similares com glyphosate nas doses de 1.200 g a.e. ha-1 (Zobiole et al., 2010a,c), 1.680 g a.e. ha-1 (Reddy et al., 2000) e 6.300 g a.e. ha ${ }^{-1}$ (King et al., 2001) descrevem reduções na biomassa seca da parte aérea e da raiz da soja RR. Considerando as doses de glyphosate atualmente recomendadas para uso na soja RR (400 a 1.200 g e.a. ha-1) no Brasil (Gazziero et al., 2008) e também doses superiores às da recomendação adotada neste experimento ( $1.800 \mathrm{~g}$ a.e. ha ${ }^{-1}$ ), não seriam esperados decréscimos nas variáveis analisadas, como observado na Figura 1, sobretudo pelo fato de o cultivar RR ser geneticamente modificado pela introdução no seu genoma do gene que codifica a EPSPS, isolado da bactéria Agrobacterium tumefaciens estirpe CP4, a qual é capaz de metabolizar o glyphosate, tornandose imune aos efeitos tóxicos desse herbicida (Padgette et al., 1996).
No segundo experimento, avaliações realizadas antes da aplicação dos tratamentos com glyphosate ou das associações de glyphosate com aminoácidos (estádio V3 - 22 DAE) demonstraram não haver diferença entre os tratamentos, os quais apresentaram praticamente os mesmos valores dos parâmetros fotossintéticos analisados pelo IRGA e da fluorescência da clorofila analisada pelo fluorômetro (Tabela 1).

Na primeira avaliação após a aplicação dos tratamentos com ou sem glyphosate e uso de aminoácidos (V7, 38 DAE), o tratamento sem glyphosate e sem aplicação de aminoácido (T1) apresentou reduções significativas em relação aos demais no que se refere aos parâmetros fotossintéticos avaliados, mostrando também valores inferiores aos da isolinha parental quando comparados pelo teste de Dunnet a 5\% de probabilidade (Tabela 2). Como a fluorescência da clorofila é uma medida que mostra o grau de eficiência com que as plantas estão fotossintetizando, essa variável pode ser usada como uma ferramenta para estudar diversos aspectos da fotossintese, pois reflete a mudança na membrana e na organização do tilacoide (Maxwell \& Johnson, 2000). Assim, T1 afetou diretamente a estrutura do cloroplasto, com significativa redução da relação $\mathrm{Fv} / \mathrm{Fm}$ (Tabela 2). Mudanças na fluorescência foram observadas em plantas tratadas com herbicidas inibidores da síntese de aminoácidos (Ireland et al., 1986) ou da rota respiratória (Gonzalez-Moro et al., 1997).

De modo geral, a adição de aminoácidos junto ou após a aplicação de glyphoaste (T2 e T3) mostrou valores dos parâmetros fotossintéticos superiores em relação aos observados em T1 (Tabela 2). Como a energia luminosa usada para fotossintese pode ser dissipada como calor, ou reemitida como luz em maior comprimento de onda (fluorescência) (Maxwell \& Johnson, 2000), os tratamentos T2 e T3 parecem ter prevenido o dano direto causado pelo glyphosate à estrutura do cloroplasto (Reddy et al., 2004), apresentando os mesmos valores de $\mathrm{Fv} / \mathrm{Fm}$ em relação à sua isolinha parental não RR (T6). Para Horton et al. (1996), diminuições em $\mathrm{Fv} / \mathrm{Fm}$ estão associadas com o aumento da excitação de energia no fotossistema II, e essa diminuição é geralmente considerada um dos indicativos de baixo fluxo 

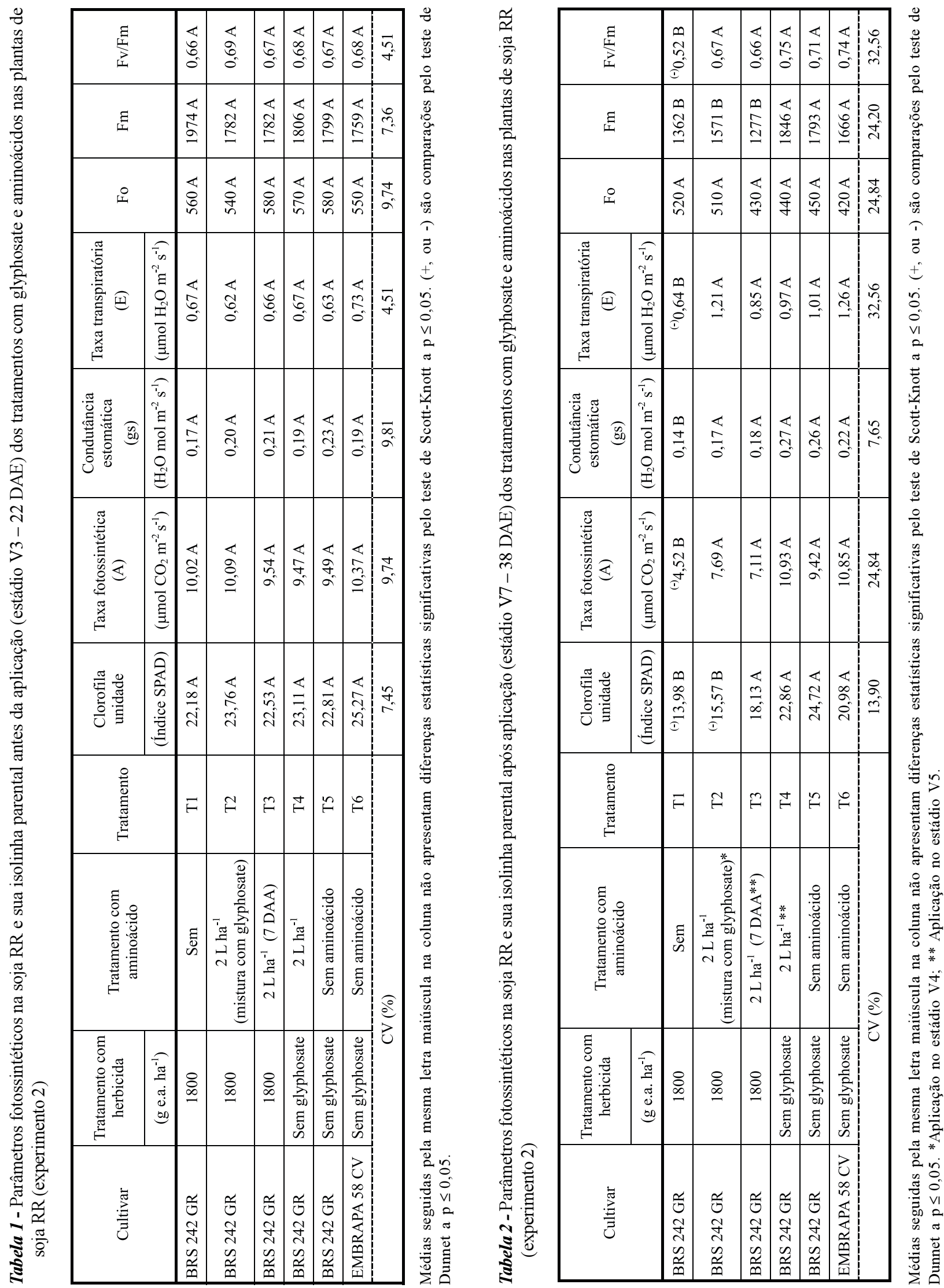
no transporte de elétron, o que se reflete em uma menor taxa fotossintética (Tabela 2).

Entretanto, os tratamentos sem aplicação de glyphosate e com ou sem adição de aminoácido (T4 e T5) não apresentaram diferenças entre eles e em relacão à sua isolinha parental não RR (T6), ou seja, a aplicação de aminoácidos não incrementou os parâmetros fotossintéticos na planta. Provavelmente, a adição de fontes de carbono (aminoácidos) no sistema-planta não levou ao aumento de fotossintese e da biomassa, pelo fato de grande parte do carbono fixado pelas plantas superiores ser sintetizada em aminoácidos aromáticos, vitaminas, lignina, alcaloides e compostos fenólicos (Kishore \& Shah, 1988).

Os mesmos resultados observados para os tratamentos sem glyphosate e com adição de aminoácidos foram também obtidos na avaliação realizada no estádio $R 1$, não se observando aumento da fotossintese com a utilização dos tratamentos com aminoácidos (Tabela 3). De acordo com o conceito do nivel de suficiência, existem niveis definidos, por exemplo, para os nutrientes individuais no solo. Segundo esse conceito, abaixo desse nivel as culturas responderão aos fertilizantes adicionados, e acima, a cultura provavelmente não responderá (Eckert, 1987). É possivel que um conceito semelhante seja válido para a aplicação exógena de aminoácidos.

$\mathrm{O}$ efeito mais pronunciado do glyphosate ocorreu na parte aérea (Tabela 3), possivelmente em função do limitado período de tempo que esse cultivar dispõe para recuperar-se, uma vez que pertence ao grupo de maturação precoce. Efeitos negativos no acúmulo de biomassa ocorrem provavelmente devido aos efeitos aditivos da diminuição da taxa fotossintética (Tabela 2) e da redução na concentração dos nutrientes na parte aérea (Zobiole et al., 2010a). Esses resultados também encontram respaldo nos trabalhos desenvolvidos por Bott et al. (2008), os quais observaram que a aplicação do glyphosate na soja RR reduziu significativamente a biomassa e a elongação da raiz.

Apesar de os tratamentos com aminoácidos e glyphosate (T2 e T3) apresentarem valores significativamente inferiores a T4, T5 e T6 para as variáveis altura, MSPA, MSRA e

\begin{tabular}{|c|c|c|c|c|c|c|c|c|}
\hline 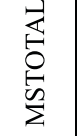 & \multirow{3}{*}{ 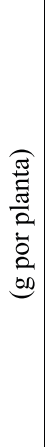 } & $\begin{array}{l}0 \\
\dot{J} \\
\tilde{5}\end{array}$ & $\begin{array}{l}m \\
\bar{\sigma} \\
\hat{\sim}\end{array}$ & $\begin{array}{l}\infty \\
\stackrel{n}{i} \\
\hat{\sigma}\end{array}$ & $\begin{array}{l}\overleftrightarrow{8} \\
\infty \\
\text { m }\end{array}$ & 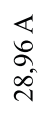 & $\begin{array}{l}\varangle \\
\bar{\sigma} \\
\infty \\
\infty \\
\text { n. }\end{array}$ & $\begin{array}{l}\text { ते } \\
\text { ते }\end{array}$ \\
\hline$\underset{\Sigma}{\mathbb{2}}$ & & $\begin{array}{l}m \\
m \\
m\end{array}$ & 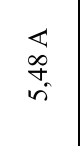 & $\begin{array}{l}\varangle \\
\text { os } \\
\stackrel{+}{*}\end{array}$ & $\begin{array}{l}\varangle \\
8 \\
0 \\
0\end{array}$ & $\begin{array}{l}\varangle \\
\hat{n} \\
\forall\end{array}$ & $\begin{array}{l}\varangle \\
a \\
n \\
6\end{array}$ & $\stackrel{\circ}{\stackrel{m}{m}}$ \\
\hline 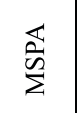 & & 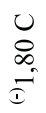 & 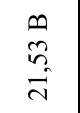 & $\begin{array}{l}\infty \\
\text { ô } \\
\text { ì }\end{array}$ & $\frac{\pi}{\pi}$ & 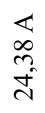 & 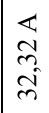 & $\begin{array}{l}\bar{\sigma} \\
\stackrel{0}{n}\end{array}$ \\
\hline 龸 & 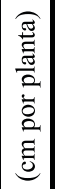 & \begin{tabular}{l}
0 \\
8 \\
$\stackrel{8}{9}$ \\
\multirow{I}{*}{}
\end{tabular} & $\begin{array}{l}n \\
8 \\
\dot{8}\end{array}$ & $\begin{array}{l}n \\
0 \\
\text { ñ } \\
\text { nิ }\end{array}$ & $\begin{array}{l}\ll \\
\stackrel{2}{2} \\
\stackrel{2}{1}\end{array}$ & $\begin{array}{l}\varangle \\
0 \\
\infty \\
\infty \\
\infty \\
0\end{array}$ & $\begin{array}{l}m \\
8 \\
i \\
i \\
6\end{array}$ & $\begin{array}{l}\hat{\infty} \\
\infty\end{array}$ \\
\hline \multicolumn{2}{|c|}{$\underset{|c|}{\stackrel{\Xi}{د}}$} & 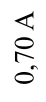 & $\frac{\pi}{\stackrel{\leftrightarrow}{0}}$ & 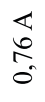 & $\frac{\ll}{\stackrel{2}{0}}$ & $\frac{\varangle}{\sigma_{0}^{\circ}}$ & $\mid \begin{array}{l}\ll \\
n \\
0 \\
0\end{array}$ & $\begin{array}{l}\hat{n} \\
\infty\end{array}$ \\
\hline \multicolumn{2}{|l|}{ ⿷匚 } & 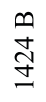 & $\begin{array}{l}\mathbb{J} \\
\stackrel{J}{I}\end{array}$ & $\begin{array}{l}\underset{I}{~} \\
\underset{I}{J}\end{array}$ & 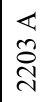 & $\begin{array}{l}\varangle \\
\frac{1}{6} \\
-1\end{array}$ & 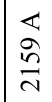 & m. \\
\hline \multicolumn{2}{|l|}{ 오 } & 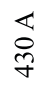 & 岁 & ঝ & $\begin{array}{l}\varangle \\
0 \\
\infty \\
+\end{array}$ & 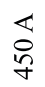 & $\begin{array}{l}\ll \\
\text { ஸn }\end{array}$ & $\begin{array}{l}n \\
\cong\end{array}$ \\
\hline 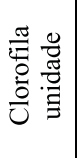 & 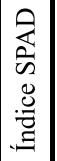 & 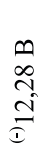 & $\begin{array}{l}0 \\
i \\
\stackrel{n}{1} \\
\stackrel{1}{i}\end{array}$ & 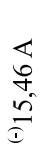 & 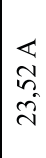 & $\underset{i}{\stackrel{\Delta}{i}}$ & 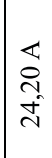 & \begin{tabular}{l}
$\curvearrowleft$ \\
\multirow{\Xi}{*}{}
\end{tabular} \\
\hline \multicolumn{2}{|c|}{ 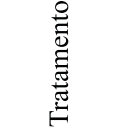 } & $\bar{F}$ & $\tilde{F}$ & $\hat{\theta}$ & $\stackrel{\nabla}{E}$ & 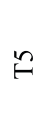 & $\bullet$ & \\
\hline \multicolumn{2}{|c|}{ 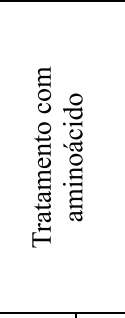 } & घี & 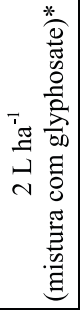 & 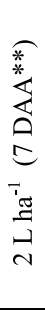 & 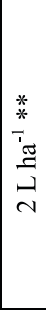 & 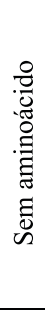 & 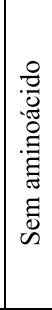 & de \\
\hline 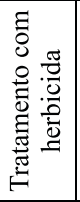 & 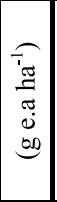 & $\stackrel{8}{\stackrel{\infty}{\infty}}$ & $\underset{\infty}{\stackrel{\infty}{-}}$ & $\underset{\infty}{\stackrel{\infty}{\infty}}$ & 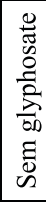 & 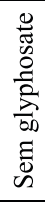 & 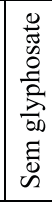 & $\vec{u}$ \\
\hline \multicolumn{2}{|l|}{ 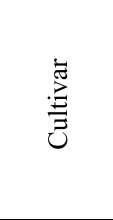 } & 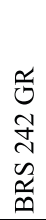 & 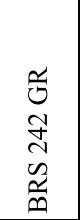 & 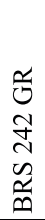 & 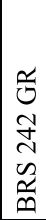 & 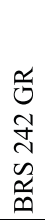 & 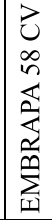 & \\
\hline
\end{tabular}


MSTOTAL (Tabela 3), o uso de aminoácidos em mistura ou após aplicação do glyphosate preveniu as injúrias causadas por esse herbicida na soja RR (T1). Comparando-se T1 com T2 e T3, o glyphosate reduziu aproximadamente em $92 \%$ a MSPA, em $35 \%$ a MSRA e em $81 \%$ a MSTOTAL. Uma das hipóteses para as reduções ocorridas no acúmulo de biomassa seca da parte aérea e da raiz em soja RR na presença do glyphosate, como discutido anteriormente, é o processo de degradação desse herbicida dentro da planta, que resulta na formação do AMPA, conhecida fitotoxina (Duke et al., 2003; Reddy et al., 2004), e provavelmente das reduções na taxa de fotossíntese, na conversão de fotoassimilados (Zobiole et al., 2010a) e na biodisponibilidade de nutrientes nos tecidos foliares (Cakmak et al., 2009; Zobiole et al., 2010a).

Kremer \& Means (2009) também observaram que o glyphosate em soja $R R$ provocou desbalanço de ácido indol acético (AIA). Como o produto comercial possui em sua composição o aminoácido triptofano, o qual é um precursor do hormônio AIA (Taiz \& Zeiger, 1998), provavelmente esse seja um dos responsáveis pelos efeitos positivos do suprimento exógeno de aminoácidos observados neste experimento. A absorção dos aminoácidos exógenos, e consequente entrada de carbono no sistemaplanta, provavelmente repercutiu na recuperação dos efeitos provocados pelo glyphosate, com consequente aumento de A, Fv/Fm (Tabela 2) e da conversão de fotoassimilados (Tabela 3).

Há evidências de que o fornecimento de fontes de nitrogênio com a aplicação de glyphosate aumenta a injúria das plantas. Reddy \& Zablotowicz (2003) avaliaram diferentes sais de glyphosate, como disopropylamine, trimethylsulfonium (Tms) e diammonium aminomethanamide dihydrogen tetraoxosulfate (Adt), e concluíram que o glyphosate-Adt causou maior injúria à soja RR, apresentando necrose com poucas horas após aplicação, além de redução do número de nódulos, leghemoglobina, peso fresco e clorofila. Bernards et al. (2005) e Mueller et al. (2006) também observaram que a adição de sulfato de amônio aumentou a porcentagem de absorção e translocação de glyphosate, com consequente aumento de efeito no controle de plantas daninhas. Em relação ao efeito da aplicação de potássio junto com glyphosate, não há até o momento evidências de que possa haver antagonismo ou sinergismo da ação do herbicida. Dessa forma, a reversão dos sintomas de fitointoxicação na soja RR, provavelmente, decorre do suprimento exógeno de aminoácidos, e não do suprimento extra de $\mathrm{N}$ e K contido no produto.

Em resumo, as variáveis fotossintéticas e a biomassa seca da parte aérea e da raiz foram afetadas pela aplicação do glyphosate, porém o segundo experimento evidenciou, de modo geral, que o uso de aminoácidos exógenos pode ser uma estratégia para prevenir os efeitos indesejáveis destes herbicida na cultura da soja RR.

\section{AGRADECIMENTOS}

Ao Conselho Nacional de Desenvolvimento Científico e Tecnológico (CNPq), pela bolsa de estudos e pelo apoio financeiro. As marcas comerciais no texto são apenas informativas e não representam nenhuma recomendação oficial da USDA-ARS.

\section{LITERATURA CITADA}

BEALE, S. I. ä-Aminolevulinic acid in plants: its biosynthesis, regulation and role in plastid development. Ann. Rev. Plant Physiol., v. 29, n. 1, p. 95-120, 1978.

BERNARDS, M. L. et al. Glyphosate interaction with manganese in tank mixtures and its effects on glyphosate absorption and translocation.Weed Sci., v. 53, n. 6, p. 787-794, 2005.

BOTT, S. et al. Glyphosate-induced impairment of plant growth and micronutrient status in glyphosate-resistant soybean (Glycine $\max$ L.). Plant Soil, v. 312, n. 1, p. 185194, 2008.

CAKMAK, I. Glyphosate reduced seed and leaf concentrations of calcium, manganese, magnesium and iron in non-glyphosate resistant soybean. Europ. J. Agron., v. 31, n. 1, p. 114-119, 2009.

CHEN, S. K.; SUBLER, S.; EDWARDS, C. A. Effects of agricultural biostimulants on soil microbial activity and nitrogen dynamics. Appl. Soil Ecol., v. 19, n. 3, p. 249-259, 2002.

DUKE, S. O. et al. Isoflavone, glyphosate, and aminomethylphosphonic acid levels in seeds of glyphosatetreated, glyphosate-resistant soybean. J. Agric. Food Chem., v. 51, n. 1, p. 340-344, 2003.

Planta Daninha, Viçosa-MG, v. 29, n. 1, p. 195-205, 2011 
ECKERT, D. J. Soil test interpretations: Basic cation saturation ratios and sufficiency levels. In: BROWN, J. R. Soil testing: sampling, correlation, calibration, and interpretation. Madison: SSSA, 1987. p. 53-64. (SSSA Spec. Publ., 21)

FERREIRA, D. F. Sistema de análise de variância (Sisvar) versão 4.6. Lavras: Universidade Federal de Lavras, 1999. CD-ROM.

FORLANI, G. Mode of action of herbicidal derivatives of aminomethylenebisphosphonic acid. Part II. Reversal of herbicidal action by aromatic amino acids. J. Plant Growth Reg., v. 16, n. 1, p. 147-152, 1997.

FRANZ, J. E.; MAO, M. K.; SIKORSKI, J. A. Glyphosate: a unique global herbicide. Washington: American Chemical Society, 1997. (ACS Monograph, 189)

GAZZIERO, D. L. P.; ADEGAS, F.; VOLL, E. Glifosate e soja transgênica. Londrina: Embrapa Soja, 2008. p. 4 (Circular Técnica 60)

GENTY, B.; BRIANTAIS, J. M.; BAKER, N. R. The relationship between the quantum yield of photosynthetic electron transport and quenching of chlorophyll

fluorescence. Biochim. Biophys. Acta., v. 990, n. 1, p. 87-92, 1989.

GONZALEZ-MORO, B. et al. Comparative effects of PPT and AOA on photosynthesis and fluorescence chlorophyll parameters in Zea mays. J. Plant Physiol., v. 151, n. 6, p. 641-648, 1997.

GRESSHOFF, P. M. Growth inhibition by glyphosate and reversal of its action by phenylalanine and tyrosine. Aust. J. Plant Physiol., v. 6, n. 1, p. 177-85, 1979.

HOAGLAND, D. R.; ARNON, D. J. The water culture method of growing plants without soil. Berkeley: University of California, 1950. $32 \mathrm{p}$.

HORTON, P.; RUBAN, A. V.; WALTERS, R. G. Regulation of light harvesting in green plants. Ann. Rev. Plant Physiol. Plant Molec. Biol., v. 4, n. 1, p. 655-684, 1996.

HUBER, D. M. Strategies to ameliorate glyphosate immobilization of manganese and its impact on the rhizosphere and disease. In: LORENZ, N.; DICK, R. Proceedings of the Glyphosate Potassium Symposium 2006. Ohio State University, AG Spectrum, DeWitt, Iowa, 2006.

IRELAND, C. R.; PERCIVAL1, M. P.; BAKER, N. R. Modification of the induction of photosynthesis in wheat by glyphosate, an inhibitor of amino acid metabolism. J. Exper. Bot., v. 37, n. 176, p. 299-308, 1986.
JAWORSKI, E. G. Mode of action of N-phosphonomethylglycine: inhibition of aromatic amino acid biosynthesis. J. Agri. Food Chem., v. 20, n. 6, p. 1195-1198, 1972.

JOHAL, G. S.; HUBER, D. M. Glyphosate effects on disease and disease resistance in plants. Europ. J. Agron., v. 31, n. 3, p. $144-152,2009$.

KABACHNIK, M. I. et al. Organophosphorus complexones. Russian Chem. Rev., v. 43, n. 9, p. 733-744, 1974.

KING, A. C.; PURCELL, L. C.; VORIES, E. D. Plant growth and nitrogenase activity of glyphosate-tolerant soybean in response to glyphosate applications. Agron. J., v. 93, n. 1, p. 179-186, 2001.

KISHORE, G. M.; SHAH, D. M. Amino acid biosynthesis inhibitors as herbicides. Ann. Rev. Biochem., v. 57, n. 1, p. 627-663, 1988.

KITCHEN, L. M.; WITT, W. W.; RIECK, C. E. Inhibition of chlorophyll accumulation by glyphosate. Weed Sci., v. 29, p. 513-516, 1981.

KREMER, R. J.; MEANS, N. E. Glyphosate and glyphosate-resistant crop interactions with rhizosphere microorganisms. Eur. J. Agron., v. 31, n. 1, p. 153-161, 2009

LEE, T. T. Effects of glyphosate on synthesis and degradation of chlorophyll in soybean and tobacco cells. Weed Res., v. 21, n. 1, p. $161-164,1981$

LIU, F. et al. Stomatal control and water use efficiency of soybean (Glycine max L. Merr.) during progressive soil drying. Environ. Exper. Bot., v. 54, n. 1, p. 33-40, 2005

MAGALHÃES FILHO, J. R. et al. Deficiência hídrica, trocas gasosas e crescimento de raízes em laranjeira "Valência" sobre dois tipos de porta enxerto. Bragantia, v. 67, n. 1, p. 75-82, 2008.

MAXWELL, K.; JOHNSON, G. N. Chlorophyll fluorescence - A practical guide. J. Exper. Bot., v. 51, n. 345, p. 659-668, 2000 .

MOORMAN, T. B. et al. Production of hydroxybenzoic acids by Bradyrhizobium japonicum strains after treatment with glyphosate. J. Agric. Food. Chem., v. 40, n. 1, p. 289-293, 1992.

MUELLER, T. C. et al. Comparison of glyphosate salts (isopropylamine, diammonium and potassium) calcium magnesium concentrations on the control various weeds. Weed Technol., v. 20, n. 1, p. 164-171, 2006

PADGETTE, S. R. et al. New weed control opportunities: development of soybeans with a roundup ready gene. In DUKE, S. O (Ed.). Herbicide resistante crops: agricultural, economic, environmental, regulatory and technological aspects. Boca Raton: CRC Press, 1996. p. 53-84. 
PROCÓPIO, S. O. et al. Características físiológicas das culturas de soja e feijão e de três espécies de plantas daninhas Planta Daninha, v. 22, n. 2, p. 211-216, 2004.

RAY, T. B. Inhibition of valine and isoleucine biosynthesis in plants. Plant Physiol., v. 75, n. 3, p. 827-831, 1984.

REDDY, K. N.; HOAGLAND, R. E.; ZABLOTOWICZ, R. $\mathrm{M}$. Effect of glyphosate on growth, chlorophyll content and nodulation in glyphosate-resistant soybeans (Glycine max) varieties. J. New Seeds., v. 2, n. 1, p. 37-52, 2000.

REDDY, K. N.; ZABLOTOWICZ, R. M. Glyphosateresistant soybean response to various salts of glyphosate and glyphosate accumulation in soybean nodules. Weed Sci., v. 51, n. 4 , p. 496-502, 2003

REDDY, K. N.; RIMANDO, A. M.; DUKE, S. O. Aminomethylphosphonic acid, a metabolite of glyphosate, causes injury in glyphosate-treated, glyphosate-resistant soybean. J. Agric. Food Chem., v. 52, n. 16, p. 5139-5143, 2004.

SANTOS, J. B. et al. Tolerance of Bradyrhizobium strains to glyphosate formulations. Crop Protec., v. 24, n. 6, p. 543-547, 2005.

SAS Institute. SAS/STAT User Guide. Version 8.2. Cary: 2001.

SHANER, D. L. et al. Physiological responses of corn to Arsenal. Proc. South Weed Sci., v. 37, p. 364, 1984.

SHIBLES, R. M.; WEBER, C. R. Leaf area, solar radiation interception, and dry matter production by various soybean planting patterns. Crop Sci., v. 5, n. 6, p. 575-577, 1965.
SPSS, 2000. SysStat (C) for Windows, Version 10.

SUBLER, S.; DOMINGUEZ, J.; EDWARDS, C. A. Assessing biological activity of agricultural biostimulants: bioassays for plant growth regulators in three soil additives. Comm. Soil Sci. Plant Anal., v. 29, n. 7, p. 859-866, 1998.

TAIZ, L.; ZEIGER, E. Plant physiology, Sunderland: Sinauer Associates, 1998. 719 p.

ZABLOTOWICZ, R. M.; REDDY, K. N. Impact of glyphosate on the Bradyrhizobium japonicum symbiosis with glyphosate-resistant transgenic soybean. J. Environ. Qual., v. 33, n. 3 , p. $825-831,2004$.

ZABLOTOWICZ, R. M.; REDDY, K. N. Nitrogenase activity, nitrogen content, and yield responses to glyphosate in glyphosate-resistant soybean. Crop Protec., v. 26, n. 3, p. $370-376,2007$.

ZOBIOLE, L. H. S. et al. Glyphosate reduces shoot concentration of mineral nutrients in glyphosateresistant soybeans. Plant and Soil, v. 328, n. 1, p. 57-69, 2010a.

ZOBIOLE, L. H. S. et al. Water use efficiency and photosynthesis of glyphosate-resistant soybean as affected by glyphosate. Pest. Biochem. Physiol., v. 97, n. 3, p. $182-193,2010$ b.

ZOBIOLE, L. H. S. et al. Effect of glyphosate on symbiotic N2 fixation and nickel concentration in glyphosateresistant soybeans. Appl. Soil. Ecol., v. 44, n. 1, p. 176-180, 2010c. 